\title{
La prévision du risque d'avalanches en France Bilan et perspectives
}

\author{
E. Pahaut et G. Giraud \\ Météo-France, Centre National de Recherches Météorologiques, Centre d'Etudes de la Neige
}

Chaque hiver, les avalanches sont responsables de la mort d'une trentaine de personnes dans les différents massifs français. Les victimes sont essentiellement des skieurs de randonnée et des alpinistes, des skieurs hors-pistes et, phénomène nouveau, des surfeurs.

Les accidents par avalanches dans les zones habitées ou sur les voies de circulation sont plus rares. Cependant, il arrive que certaines situations météorologiques remarquables soient à l'origine d'une activité avalancheuse préoccupante pour la sécurité des résidants en montagne. Les conséquences peuvent être catastrophiques pour l'économie montagnarde : dégâts aux forêts, installations techniques touchées, impact sur le tourisme hivernal...

Le bilan de ces vingt dernières années montre que la hausse spectaculaire de la fréquentation de la montagne n'a pas entraîné d'augmentation sensible du nombre d'accidents par avalanche. Les mesures de prévention qui ont été progressivement mises en œuvre ont, semble-t-il, prouvé une certaine efficacité.

La prévention fait appel à deux types d'actions. La gestion spatiale du risque d'avalanche consiste à mettre en œuvre des moyens de protection, actifs ou passifs, utilisant toutes les techniques du génie paravalanche. Les secteurs sensibles sont identifiés, le risque évalué et les mesures de protections les plus appropriées sont décidées.

La Prévision du Risque d'Avalanche (PRA) à laquelle nous nous intéressons ici, relève de la gestion temporelle du risque. Elle nécessite un dispositif capable d'assurer la surveillance du manteau neigeux et de prévoir son évolution en fonction des conditions météorologiques. La diffusion de bulletins réguliers ou spéciaux permet d'informer les usagers, d'alerter le cas échéant les services de sécurité qui prendront alors les mesures temporaires utiles.

L'organisation actuelle de la Prévision du Risque d'Avalanche (Pahaut [1]) repose sur un partenariat entre les stations de sports d'hiver et Météo-France. Aux stations la mission d'assurer les observations, à Météo-France d'assurer la prévision et la diffusion de l'information « neige et avalanche ».

Entre 1970 et 1987, la PRA est assurée par le CEN sur l'ensemble des massifs montagneux français. Depuis 1987 , cette assistance est confiée aux centres météorologiques des départements alpins, pyrénéens et corses. Chacun de ces centres est compétent sur un ou deux départements. La PRA ainsi décentralisée rapproche les prévisionnistes du terrain et facilite les contacts avec les responsables locaux.

L'information « neige et avalanche " est délivrée par le canal de bulletins départementaux. Chaque département a été découpé en zones de quelques centaines de kilomè- tres carrés : 23 pour les Alpes, 11 pour les Pyrénées et 2 pour la Corse. C'est à l'échelle de la zone que sont généralement indiquées les informations sur les conditions de neige et de risque.

\section{QUELLE PRÉVISION DE RISQUE D'AVALANCHE?}

Le risque d'avalanche n'est pas un paramètre mesurable. Si l'on ne sait prévoir l'avalanche, l'endroit et l'heure de son déclenchement, le phénomène avalanche ne procède pas du seul hasard. Les conditions favorables aux déclenchements spontanés d'avalanches sont relativement bien identifiées et le risque est évalué avec une bonne probabilité de réussite.

Pour ce qui concerne les déclenchements provoqués, de type accidentel, les progrès ont été moins nets. Beaucoup de lacunes subsistent encore dans la connaissance des paramètres pertinents à l'origine de ce type de déclenchements. L'action du vent est reconnue mais insuffisamment expliquée.

Quels sont les facteurs qui expliquent les différentes prises de cohésion de la neige ? Pourquoi les fractures dans le manteau neigeux intéressent-elles un matériau qui peut se montrer pulvérulent ou, au contraire, dur et cassant ? Quel est le rôle de la température, de la teneur en liquide, de la nature des cristaux de neige ?

Beaucoup de réponses ne sont que de simples hypothèses. Des campagnes de mesures sont actuellement en cours pour tenter de comprendre certains comportements du matériau « neige ». Ces expériences restent cependant assez lourdes et délicates à mener car elles nécessitent une présence quasi permanente sur le terrain (Duclos [2]).

Malgré toutes ces difficultés, la Prévision du Risque d'Avalanche se doit cependant de répondre à un certain nombre de questions.

Quelle est la probabilité de déclenchement? Quelle est le type d'avalanche que l'on doit craindre ? Quelles sont les zones concernées ?

On sait que le déclenchement d'une avalanche résulte de I'interaction de nombreux paramètres : paramètres internes comme le type de grains, qualité des liaisons entre les strates, profils verticaux de température, de densité, de teneur en eau liquide, de résistance à l'enfoncement... Paramètres de surface comme l'épaisseur et la qualité de la neige récente, effets du vent, humidification de la couche superficielle... 
La stabilité du manteau neigeux et le risque d'avalanche sont donc liés à l'action conjuguée de plusieurs facteurs qui vont évoluer, en un point donné, en fonction des seules conditions météorologiques.

Certaines avalanches peuvent être qualifiées de «météorologiques ». Elles sont directement liées à des conditions météorologiques remarquables, sinon violentes. Elles se produisent pendant ou immédiatement après d'abondantes chutes de neige, après un réchauffement très marqué ou encore au cours d'une période particulièrement ventée.

Une deuxième catégorie d'avalanches traduit l'effet «mémoire» du manteau neigeux. Les grands traits de l'hiver sont inscrits dans les caractéristiques des différentes strates. Le déclenchement d'une avalanche peut très bien s'expliquer par des conditions météorologiques datant de plusieurs semaines, ayant entraîné une métamorphose ou créé une liaison entre strates défavorables à la stabilité du manteau neigeux. C'est bien souvent cette deuxième catégorie d'avalanches qui est à l'origine des accidents provoqués en hors piste ou en randonnée.

Pour être efficace, un système de prévision du risque d'avalanche doit pouvoir observer le manteau neigeux. C'est sur le schéma "observer, analyser, prévoir" que s'appuie le dispositif opérationnel mis en place en France après la série d'avalanches meurtrières de l'hiver 1970.

\section{II 口 OBSERVER -}

C'est la mission du réseau nivo-météorologique. Ce réseau de mesures, exploité dans le cadre d'un partenariat entre les stations de sports d'hiver et Météo-France, comprend près de 140 postes de mesure répartis sur les Alpes, les Pyrénées et la Corse. Deux séries d'observations sont réalisées tous les jours en début de matinée et en mijournée. Nuages, vent, températures et précipitations, épaisseur et qualité de la neige fraîche, hauteur totale de la neige au sol, phénomènes de chasse-neige sur les crêtes, avalanches observées sont l'objet de relevés précis. Toutes ces informations sont ensuite codifiées et transmises au Centre Météorologique Départemental, chargé de la PRA. La majorité des postes de mesures sont situés dans la tranche d'altitude $1500 / 2000 \mathrm{~m}$.

Les sondages par battage et les profils stratigraphiques sont des mesures pratiquées dans le manteau neigeux. Ce sont les seules mesures qui permettent d'identifier les différentes strates. La connaissance de paramètres internes comme le type de grains, la cohésion, la densité, la teneur en eau liquide est essentielle pour évaluer la stabilité du manteau neigeux. Ces investigations dans le manteau neigeux sont effectuées au moins une fois par semaine sur un grand nombre de sites, dont l'altitude est généralement comprise entre 1800 et $2500 \mathrm{~m}$.

Un réseau d'une quinzaine de stations automatiques mesurent en continu la température de l'air, la vitesse du vent, l'épaisseur de la couche de neige déposée au sol. Situées entre 2000 et $3000 \mathrm{~m}$, ces stations automatiques fournissent un complément d'informations dans des zones où l'observation humaine n'est pas possible.

\section{D ANALYSER ET PRÉVOIR}

L'apparition des modèles déterministes d'évolution du manteau neigeux sera probablement aussi importante pour la prévision du risque d'avalanche que la mise en exploitation des modèles météorologiques a pu l'être pour la prévision du temps.

Aussi, deux périodes seront distinguées dans les méthodes employées pour l'analyse et la prévision du risque d'avalanche : avant les modèles..., avec les modèles...

\subsection{Avant les modèles...}

Dans le cadre du dispositif national mis en place en 1970 , cette mission est confiée aux Centres Météorologiques Départementaux, chargés de la PRA. Les prévisionnistes « avalanches » collectent par télécopie ou téléphone les messages provenant des postes de leur département. Une application informatique "ADIPRA " assure toutes les fonctions de saisie, de contrôle, de visualisation des données. Les représentations synthétiques des différents paramètres sous forme de tableaux, de graphiques ou de cartes facilitent la tâche d'analyse.

L'examen des sondages par battage et des profils stratigraphiques s'avère être un des éléments déterminants du diagnostic du prévisionniste.

Ce dernier attache également beaucoup d'importance au contact direct avec l'observateur. Des renseignements occasionnels ne pouvant être intégrés à l'observation standard sont ainsi échangés et viennent parfois compléter et affiner l'information disponible.

La pratique de la montagne est un atout supplémentaire pour le prévisionniste. Ses propres observations sur le terrain sont une source appréciable de renseignements sur l'état de la neige. Elles lui permettent de répondre à certaines interrogations, qui ne peuvent trouver de réponse dans les seules données du réseau nivo-météorologique.

Après le phase de l'informatisation du traitement des données nivo-météorologiques en 1983, d'autres outils ont été mis à la disposition du prévisionniste. C'est ainsi que des modèles statistiques basés sur l'analyse discriminante ou sur la recherche de journées analogues (Obled [3] et Navarre [4]) leur ont apporté une aide efficace. Ce type de modèle développé pour des besoins locaux, à l'échelle d'un domaine skiable, a été ensuite adapté à l'échelle du massif, plus proche des préoccupations du prévisionniste départemental.

Ces modèles se sont malheureusement tous heurtés à la difficulté de prendre en compte la structure interne du manteau neigeux, pourtant essentielle pour l'évaluation de la stabilité et des risques d'avalanches. Les exigences de taille et de qualité des fichiers utilisés par ces modèles ont également limité leur implantation sur de nombreux sites ou massifs.

L'art du prévisionniste consiste donc, à partir de l'analyse de données fournies par des points de mesures, à identifier le type et le niveau de l'instabilité pour en déduire le risque existant. Puis, il doit évaluer l'incidence des conditions météorologiques prévues sur le manteau neigeux. Le prévisionniste doit user également de son esprit de synthèse. Partant d'un ensemble de données ponctuelles, il restitue les particularités les plus représentatives à l'échelle d'un massif.

On le voit, la démarche du prévisionniste relève de l'expertise. Sa mémoire, parfois secondée par des modèles basés sur la recherche de journées analogues, est fortement et constamment sollicitée. 
La Prévision du Risque d'Avalanche à l'échelle du département qui vient d'être décrite, a été conçue par les services de l'Etat avec le concours de collectivités locales et des communes de montagne. Un autre niveau de prévision du risque d'avalanche existe au niveau local. Cette échelle de travail est encore peu répandue mais elle devrait se développer au cours des prochaines années. Parallèlement à leur utilisation par les centres départementaux de Météo-France, les données nivo-météorologiques ont vocation a être utilisées localement, par les services des pistes et de la sécurité des stations de sports d'hiver. Elles sont d'une grande utilité pour la gestion du domaine skiable. Certaines stations, conscientes de l'importance que prend l'information "sécurité " des skieurs, ont reproduit, à l'échelle du domaine skiable, un dispositif similaire à celui existant à l'échelle du massif. A partir des mesures effectuées le matin en plusieurs points de son domaine, une prévision du risque valable pour la journée est réalisée et affichée à l'intention des usagers fréquentant la station.

Météo-France met à la disposition des postes nivométéorologiques un logiciel de saisie et de visualisation. Le logiciel «GELINIV » (Dumas [5]) offre les mêmes fonctionnalités que l'application départementale «ADIPRA ». Des modèles locaux d'aide à la prévision du risque d'avalanche sont aussi proposés aux services des pistes. Ces modèles statistiques sont basés sur la recherche de journées analogues. La journée analysée est caractérisée par un certain nombre de paramètres. Elle est ensuite comparée aux journées du passé. Les journées les plus proches sont sélectionnées et sont caractérisées par une activité avalancheuse. Le prévisionniste local dispose donc d'un outil supplémentaire pour estimer le caractère avalancheux de la journée considérée. Le modèle "ASTRAL " (Guyomarc'h [6]), basé sur ce principe, est proposé par MétéoFrance à toutes les stations du réseau qui disposent de séries de données répondant à des critères de durée et de qualité. Des opérateurs privés développent des produits similaires. «NXLOG " (Bolognesi [7]) est un modèle hybride qui combine approche statistique et connaissance experte.

\subsection{Avec les modèles...}

Jusqu'en 1990, une des faiblesses du système de surveillance est liée à la difficulté que peut éprouver le prévisionniste à suivre l'évolution du manteau neigeux dans toute sa diversité. Sur un massif donné, les conditions de neige sont très différentes suivant l'altitude, l'exposition, l'angle de pente... Les 140 postes du réseau nivométéorologique laissent supposer que la densité de points de mesure est forte. Pourtant, si l'on répartit ces points en fonction de l'altitude et de l'exposition, les mailles du réseau apparaissent alors très larges. De plus, des mesures essentielles comme le sondage par battage ne sont faites qu'une fois par semaine. Dans les cas de situations météorologiques très changeantes il devient malaisé de déterminer comment a pu évoluer le manteau neigeux. La difficulté à estimer le taux d'humidification et l'altitude à laquelle ce phénomène est encore sensible a valeur d'exemple.

Le prévisionniste doit donc procéder par extrapolations successives pour connaître, avec une marge d'erreur non négligeable, l'état de la neige hors des mailles de son réseau.
Il fallait donc imaginer et mettre au point l'outil qui permettrait au prévisionniste, en complément des informations issues du réseau de mesures, de connaître la structure interne du manteau neigeux dans sa grande variabilité.

\subsubsection{Le modèle "CROCUS "}

Une première étape a été franchie avec la mise à disposition du prévisionniste d'un modèle déterministe d'évolution énergétique et morphologique du manteau neigeux, baptisé CROCUS (Brun [8]). A partir de variables purement météorologiques, ce modèle simule l'évolution en terme d'accroissement, de tassement, de densité, de teneur en eau liquide, de stratigraphie et de métamorphose. Le prévisionniste l'active manuellement sur les données d'un poste du réseau nivo-météorologique. Il dispose ainsi, entre deux sondages effectivement réalisés, de l'évolution des différents paramètres caractéristiques du manteau neigeux. Cet outil performant offre aussi la possibilité de jouer des scénarios météorologiques en prévision. Cependant, cette version locale de CROCUS, ne permet pas une couverture spatiale importante. Le nombre de simulations est en effet limité puisque le modèle est alimenté manuellement en données météorologiques.

\subsubsection{La chaîne "Safran/Crocus/Mepra "}

Pour être pleinement opérationnel et renseigner les prévisionnistes « avalanches » sur l'état du manteau neigeux en de nombreuses pentes, il fallait développer un système capable de fournir à CROCUS automatiquement et en temps réel les variables météorologiques utiles. C'est le rôle de SAFRAN (Durand [9]) : Système d'Analyse Fournissant des Renseignements Atmosphériques pour la Neige.

SAFRAN utilise les sorties des modèles météorologiques (ARPEGE, modèle français ou modèle du Centre européen), les données des réseaux météorologique et nivométéorologique ainsi que celles de stations automatiques en montagne pour estimer, sur des pentes types définies par une exposition, une altitude et une pente, les paramètres pertinents pour CROCUS. Les manteaux neigeux ainsi simulés quotidiennement par le modèle déterministe sont ensuite analysés par le système expert MEPRA (Giraud [10]), dernier maillon de la chaîne (fig. 1). Ce modèle dont l'architecture de la base de connaissance est très proche du raisonnement habituel du prévisionniste «avalanches", complète les profils CROCUS du manteau neigeux en rajoutant notamment des informations mécaniques (résistance aux cisaillements et à l'enfoncement, contrainte de gravité). Ensuite, il les analyse en terme de stabilité mécanique (type de profil supérieur et inférieur, niveaux et degrés d'instabilité...) et évalue le risque d'avalanche. Le risque de départ naturel est évalué dans une échelle à 6 niveaux (très fort à très faible) et le risque accidentel dans une échelle à 4 niveaux (faible à fort).

Une interface entre les différents modules de la chaîne et le prévisionniste a été développée. Elle offre la possibilité de visualiser graphiquement des paramètres bruts ou élaborés issus des modèles SAFRAN (Température de l'air, humidité, précipitations...), CROCUS (Hauteur de neige, Température de surface, profondeur d'humidification ou de regel...) ou MEPRA (risque et type d'avalanches naturelles, 


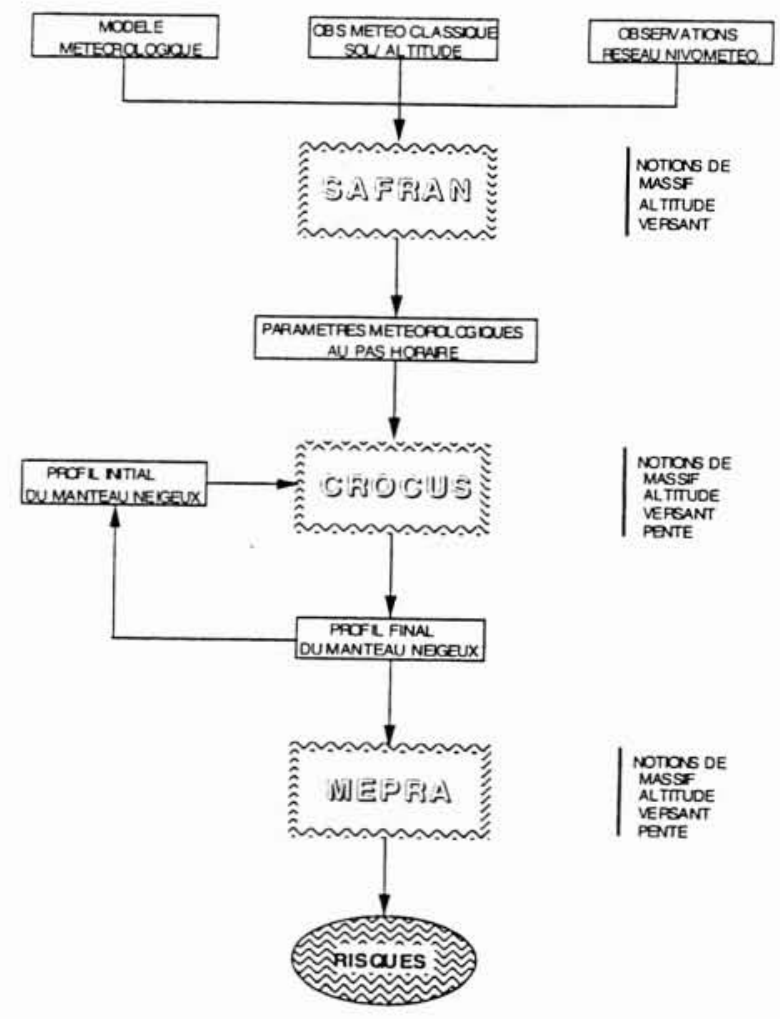

\section{Architecture de la chaîne SAFRAN/CROCUS/ MEPRA.}

risque d'avalanches accidentelles, type de profil...). Tous ces paramètres sont soit pointés sur une carte stylisée des Alpes Françaises soit visualisés sous forme de cercles concentriques (altitude, exposition) appelés «camemberts » ou projetés sur un modèle numérique de terrain avec une maille de $75 \mathrm{~m}$.

De plus, pour analyser plus finement la situation nivologique et avalancheuse, la possibilité est offerte aux prévisionnistes de visualiser :

- les profils complets du manteau neigeux par tranche d'altitude ou par exposition (fig. 2)

- I'historique des grains de neige ou de la température dans le manteau neigeux

et d'actionner une fonction "justification " du raisonnement du système expert MEPRA donnant l'évolution temporelle du risque ou le profil complet avec les niveaux d'instabilité, le type de profil supérieur et inférieur, les risques de départ naturel ou accidentel...

La chaîne SCM fonctionne du $1^{\text {er }}$ août au 31 juillet de l'année suivante, avec des conditions initiales d'enneigement fixées à zéro le $1^{\mathrm{er}}$ août. Le prévisionniste de l'Isère, département pilote, dispose ainsi chaque jour et sur chacun de ses massifs, de manteaux neigeux entièrement simulés et d'une estimation des risques d'avalanches par tranche d'altitude de $300 \mathrm{~m}$, sur 6 expositions et 2 pentes. Afin de pallier à l'impossibilité actuelle de réinitialiser cette chaîne dite de type "massif ", une chaîne de type "poste" a été mise à la disposition du prévisionniste au début de l'hiver 1994/1995. Dans cette nouvelle chaîne, les simulations ne sont plus faites sur des pentes typiques mais sur les sites où sont réalisés les sondages et profils stratigraphiques. Ces points de mesure sont caractérisés par leur appartenance à un massif et par des paramètres topographiques : altitude, exposition, pente et masques solaires. Le prévisionniste a ensuite la possibilité de comparer les manteaux neigeux réels et simulés. Le résultat de cette comparaison sera ensuite utilisé pour corriger l'analyse experte de la chaîne « massif ».

Ce produit a été très favorablement accueilli, « un peu de la même manière que l'application METEOTEL a révolutionné en son temps le travail du prévisionniste METEO " d'après Jacques Villecrose, extrait du bilan d'utilisation de la chaîne SAFRAN/CROCUS/METRA par le CDM de St Martin d'Hères saison 92-93 (Villecrose [11]).

La validation de la chaîne SCM (Martin [12] et Giraud [13]) a confirmé le bon fonctionnement global dans la tranche $900 \mathrm{~m}-3000 \mathrm{~m}$. Le prévisionniste dispose ainsi d'informations fiables sur les limites d'enneigement et l'épaisseur de neige au sol, les profondeurs d'humidification et de regel ainsi qu'une estimation correcte du risque de départ naturel d'avalanches. De plus, la chaîne SCM reste une des seules sources d'informations en début et fin de saison nivologique. Bien qu'elle souffre aussi de la rareté ou du manque de données lorsque les stations de ski sont fermées, elle fournit 'en permanence des résultats utilisables.

Avec l'arrivée d'une nouvelle génération de calculateurs, la chaîne SCM sera installée dans tous les centres météorologiques départementaux chargés de la prévision du risque d'avalanches. Les résultats seront consultables dans une application nivologique intégrée, version améliorée de l'application ADIPRA. Le prévisionniste disposera ainsi d'une imagerie unique pour visualiser les données mesurées dans le réseau et les données simulées par la chaîne.

Parallèlement, le projet météorologique SYMPOSIUM offrira à SAFRAN, en temps réel, des prévisions expertes de paramètres météorologiques par massif. Ainsi la chaîne SCM fonctionnera avec la prévision météorologique propre au département.

\section{Q QUELLES PERSPECTIVES PEUT-ON EN- VISAGER A PLUS LONG TERME ?}

Le choix de simuler le manteau neigeux et d'analyser le risque d'avalanches dans un espace altitude, exposition et pente est très adapté à une prévision à l'échelle départementale. Néanmoins, ce découpage n'est pas suffisamment fin pour prendre en compte tous les phénomènes locaux, transport de neige par le vent notamment, et donc pour décrire toute la variabilité naturelle du manteau neigeux. Le rêve du prévisionniste départemental et surtout local serait de pouvoir assurer un suivi en temps réel et très fin de certains sites avalancheux. Dans une perspective à long terme, ce rêve pourrait devenir réalité grâce au couplage de "ELSA " (Buisson [14]) avec la chaîne SCM. ELSA est actuellement un logiciel d'aide à l'analyse spatiale de sites avalancheux développé par la division nivologie du CEMAGREF. Le site avalancheux de travail est décomposé en petits panneaux, homogènes du point de vue de la forme du terrain, de la pente, de la rugosité, de l'exposition... ELSA modélise le chargement en neige de chaque panneau et leur déclenchement en jouant des scénarios 
oisans 11/01/1995 $6 \mathrm{H}$

versant: $\mathrm{N}$ pente: 40 degres
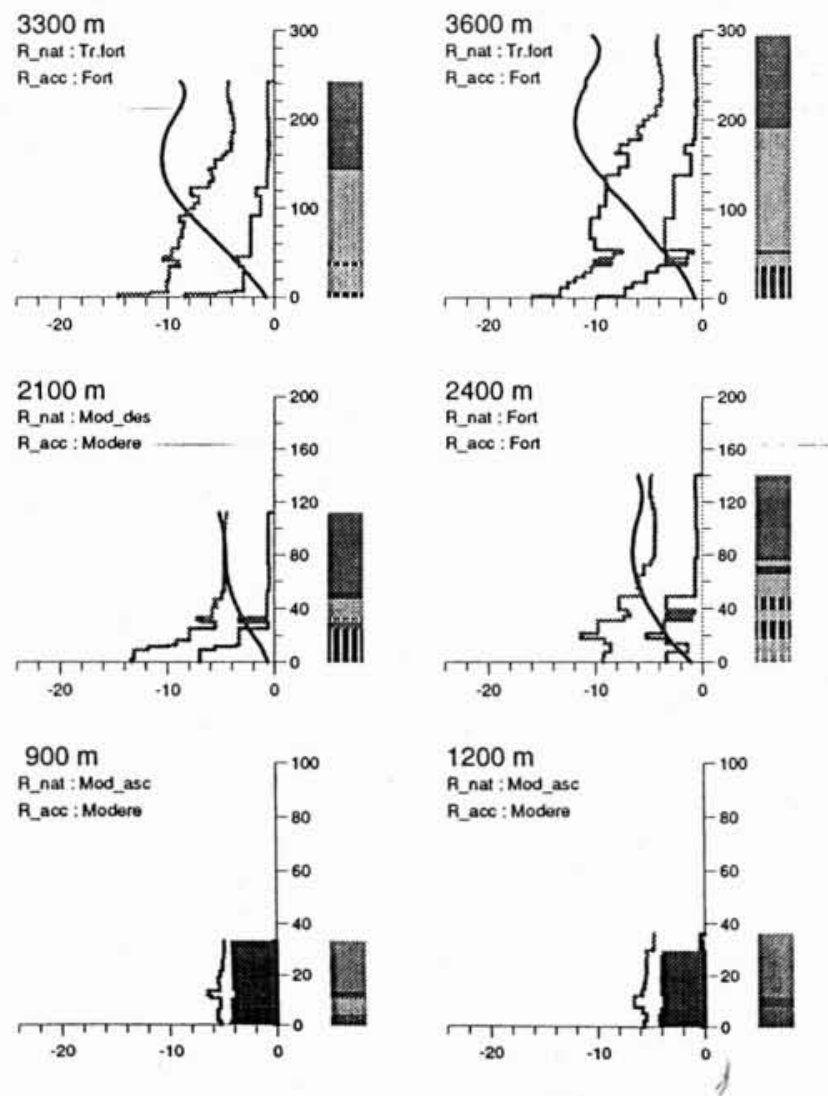
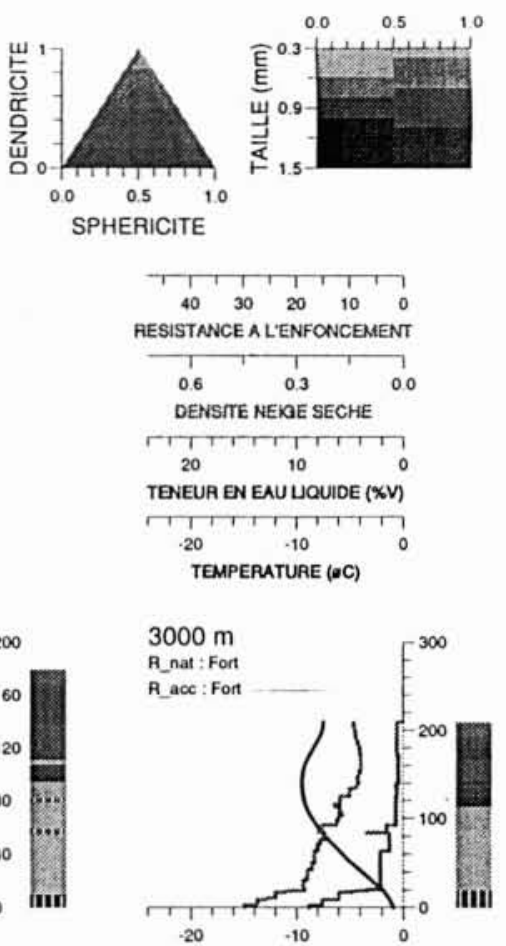

$500 \mathrm{~m}$
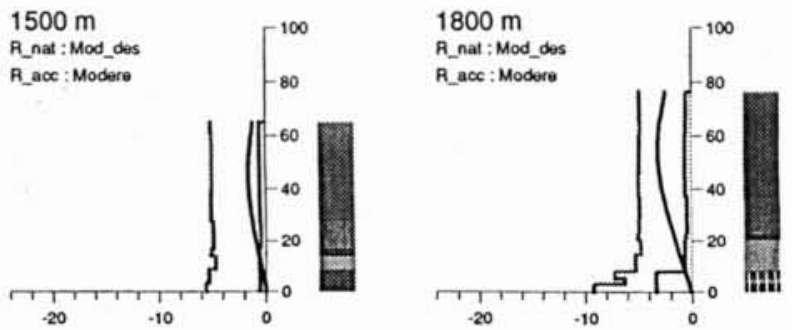

2. Massif de l'Oisans : profils du manteau neigeux simulés en versant Nord, pente à $40^{\circ}$.

nivométéorologiques choisis. La gestion spatiale du risque est le point fort d'ELSA. La gestion temporelle du risque étant le point fort de la chaîne SCM, le couplage des deux approches pourrait offrir des perspectives intéressantes pour le suivi plus détaillé de certains sites avalancheux. Une meilleure connaissance des effets locaux du transport de neige (Guyomarc'h [15]) par le vent est une des conditions préalables à la réussite de ce projet. Le prévisionniste avalanche pourrait ainsi disposer de :

- manteaux neigeux simulés et de risques d'avalanche estimés et prévus par massif montagneux à différentes orientations, pentes et altitudes avec une discrétisation de 200 ou $300 \mathrm{~m}$

— simulations détaillées sur quelques sites réels de son département.

Si au cours des 20 dernières années aucune révolution n'est venue bouleverser les méthodes utilisées en PRA opérationnelle, les modèles d'évolution du manteau neigeux et les systèmes experts devraient les modifier profondément. Avec ces capacités nouvelles de suivi dans l'espace et dans le temps, avec la possibilité d'affiner l'échelle de travail, plus que jamais les prévisionnistes de l'an 2000 auront besoin d'un esprit de synthèse particulièrement aigu...

\section{Références}

[1] Pahaut E., Brun E., Brunot G. (1991). - L'organisation de la prévision du risque d'avalanches en France, Proceedings du symposium CISA-IKAR, Chamonix, 4-8 juin 1991, p. 50-56.

[2] Duclos A. - Neige, vent et avalanches: quelques observations pour un meilleur diagnostic, revue de I'ANENA. Neige et Avalanches, n 64, décembre 1993.

[3] OBLeD C., Bots Ph.: Vers un système opérationnel de prévision numérique des avalanches à partir de méthodes statistiques, Hydrological sciences Bulletin, vol. $18, \mathrm{n}^{\circ} 4$, p. 419-429.

[4] Navarre J.-P., Guyomarc"H G., Giraud G. - Un modèle statistique pour la prévision locale, 1987, IAHS-Publ. 162, p. 571-580.

[5] DumAS J.-L., BOLOGNESI R. - GELINIV-NXLOG revue de I'ANENA, Neige et Avalanches, n 65, mars 1994. 


\section{PRÉVISION DU RISQUE D'AVALANCHES}

[6] GuYOMARC'H G., Merindol. L. - Que faut-il savoir sur ASTRAL? revue de I'ANENA, Neige et Avalanches, ${ }^{\circ} 66$, p. 21-25.

[7] Bolognesı R. - Premier dévelopement d'un modèle hybride pour le diagnostic spatial des avalanches, La Houille Blanche, $\mathrm{n}^{\circ} 8,1993$.

[8] Brun E., Martin E., Simon V., Gendre C., Coléou C. (1989). - An energy and mass model of snow cover suitable for operational avalanche forecasting, J. of Glaciol., vol. 35 , $\mathrm{n}^{\circ} 121$, p. 333-342.

[9] Durand Y., Brun E., Mérindol L., Guyomarch G., LESAFFRE B., MARTIN E. (1993). - A meteorological estimation estimation of relevant parameters for snow models, Annals of Glaciol., vol. 18, p. 65-71.

[10] GIRAUD G. (1992), - MEPRA : an expert system for avalanche risk forecasting. Proceedings of the International snow science workshop, 4-8 oct. 1992, Breckenridge, Colorado, USA, p. 97-106.
[11] VILLECROSE J. - Rapport interne sur l'utilisation de la chaîne SAFRAN/CROCUS/METRA, juin 1993.

[12] Martin E., Brun E., Durand Y. (1994). - Sensitivity of the French Alps snow cover to the variation of climatic variables, Annales Geophysicae 12, 469-477 (1994).

[13] GiRAud G. (1992). - Validation of objective models to simulate snow cover stratigraphy and avalanche risk for operational avalanche forecasting. Proceedings of the International snow science workshop, 30 oct.-3 nov. 1994. Snowbird, Utah, USA.

[14] BUisson L., ChARLeR C. - Avalanche starting zone analysis by use of a knowledge-based system, Annals of glaciology, vol. 13, 1989.

[15] Guyomarc' H G., CAstelle T. (1992). - A study of wind drift snow phenomena on an alpine site, Proceedings of the International snow science workshop, 4-8 oct. 1992, Breckenridge, Colorado, USA, p. 57-67. 